\title{
BMJ Open Association of type 2 diabetes with in- hospital complications among women undergoing breast cancer surgical procedures. A retrospective study using the Spanish National Hospital Discharge Database, 2013-2014
}

Ana Lopez-de-Andres, ${ }^{1}$ Isabel Jimenez-Trujillo, ${ }^{1}$ Valentin Hernandez-Barrera, ${ }^{1}$ Javier de Miguel-Diez, ${ }^{2}$ Manuel Mendez-Bailon, ${ }^{3}$ Jose M de Miguel-Yanes, ${ }^{4}$ Napoleon Perez-Farinos, ${ }^{5}$ Miguel Angel Salinero-fort, ${ }^{6}$ Jose L del Barrio, ${ }^{1}$ Martin Romero-Maroto, ${ }^{1}$ Rodrigo Jimenez-Garcia ${ }^{1}$

To cite: Lopez-de-Andres A, Jimenez-Trujillo I, HernandezBarrera V, et al. Association of type 2 diabetes with inhospital complications among women undergoing breast cancer surgical procedures. A retrospective study using the Spanish National Hospital Discharge Database, 2013-2014. BMJ Open 2017;7:e017676. doi:10.1136/ bmjopen-2017-017676

- Prepublication history for this paper is available online. To view these files, please visit the journal online (http://dx.doi. org/10.1136/bmjopen-2017017676).

Received 8 May 2017 Revised 6 October 2017 Accepted 13 October 2017

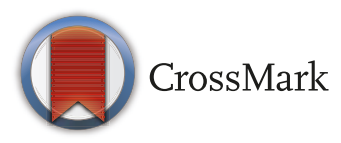

For numbered affiliations see end of article.

Correspondence to Dr Rodrigo Jimenez-Garcia; rodrigo.jimenez@urjc.es

\section{ABSTRACT}

Objectives To compare the type of surgical procedures used, comorbidities, in-hospital complications (IHC) and in-hospital outcomes between women with type 2 diabetes mellitus (T2DM) and age-matched women without diabetes who were hospitalised with breast cancer. In addition, we sought to identify factors associated with IHC in women with T2DM who had undergone surgical procedures for breast cancer.

Design Retrospective study using the National Hospital Discharge Database, 2013-2014.

Setting Spain.

Participants Women who were aged $\geq 40$ years with a primary diagnosis of breast cancer and who had undergone a surgical procedure. We grouped admissions by T2DM status. We selected one matched control for each T2DM case.

Main outcome measures The type of procedure (breast-conserving surgery (BCS) or mastectomy), clinical characteristics, complications, length of hospital stay and in-hospital mortality.

Results We identified 41458 admissions (9.23\% with T2DM). Overall, and in addition to the surgical procedure, we found that comorbidity, hypertension and obesity were more common among patients with T2DM. We also detected a higher incidence of mastectomy in women with T2DM (44.69\% vs $42.42 \%)$ and a greater rate of BCS in patients without T2DM (57.58\% vs $55.31 \%)$. Overall, non-infectious complications were more common among women with T2DM (6.40\% vs $4.56 \%)$. Among women who had undergone BCS or a mastectomy, IHC were more frequent among diabetics (5.57\% vs $3.04 \%$ and $10.60 \%$ vs $8.24 \%$, respectively). Comorbidity was significantly associated with a higher risk of IHC in women with diabetes, independent of the specific procedure used. province

Conclusions Women with T2DM who undergo surgical breast cancer procedures have more comorbidity, risk factors and advanced cancer presentations than matched
Strengths and limitations of this study

The strengths of our study include the large sample size, the standardised methodology, and the use of age-matched and province-matched surgical procedure and stage-matched women without type 2 diabetes mellitus to control for the confounding effects of these variables.

- A limitation of this study is the lack of information on chemotherapy or radiotherapy treatments prior to surgery, which may have affected our outcome variables.

- We lacked data such as specific breast cancer characteristics or accurate time frames since diagnosis.

- The association of obesity on in-hospital complications must be interpreted with caution for the possible existence of a codification bias.

patients without T2DM. Mastectomies are more common in women with T2DM. Moreover, the procedures among women with T2DM were associated with greater IHC. Comorbidity was a strong predictor of IHC in women with T2DM.

\section{INTRODUCTION}

Breast cancer is the most common type of cancer in women. ${ }^{1}$ Recently, the Global Burden of Disease Cancer Collaboration reported that the incidence of breast cancer between 2005 and 2015 in Western Europe increased by $24 \%$, from 100.6 to 124.7 cases per 100000 individuals. Breast cancer was also the leading cause of cancer-related deaths in women. ${ }^{1}$ 
Breast-conserving surgery (BCS) and mastectomy still represent the current therapeutic approach for breast cancer. Recently, the use of BCS has increased and become the primary surgical treatment for breast cancer. ${ }^{23}$ In Spain, over $80 \%$ of women diagnosed with breast cancer underwent surgery and, among these patients, nearly $75 \%$ received BCS. ${ }^{3}$ However, recent studies have shown an increased number of mastectomies in women who are candidates for BCS because of the rise of more cosmetically appealing techniques. ${ }^{4}$

The Spanish Healthcare System is public and offers universal coverage with no out-of-pocket expenses for patients.

Recently, a report using the American College of Surgeons National Surgical Quality Improvement Programme (NSQIP) database to study 30-day complication rates after breast operations concluded that, overall, BCS and mastectomy with implant reconstruction have low complication rates. Additionally, BCS in early stage breast cancer was associated with fewer overall early postoperative complications when compared with mastectomy (OR 2.8; 95\% CI 2.2 to 3.5, $\mathrm{p}<0.01) .^{5}$

Diabetes is a predictor associated with postoperative complications in women who undergo surgical procedures for breast cancer. ${ }^{5-7}$ In women with early stage breast cancer, diabetes has been found to be a risk factor for overall postoperative complications (OR 1.8; 95\% CI 1.02 to $3.4, \mathrm{p}=0.04) .^{5}$ de Blacam et al, using the NSQIP database, reported that independent risk factors for the development of an infection of any surgical wound caused by a mastectomy included a high body mass index, smoking and diabetes. ${ }^{7}$

To the best of our knowledge, no previous studies have investigated factors associated with in-hospital complications (IHC) among women with diabetes undergoing breast cancer-related surgical procedures.

Using the Spanish National Hospital Discharge Database (SNHDD) for 2013-2014, we aim in this study to i) compare variables between type 2 diabetes (T2DM) and matched women without diabetes hospitalised with breast cancer who underwent a surgical procedure, including the type of surgical procedure used (BCS or mastectomy), patient comorbidities, IHC and in-hospital outcomes (length of hospital stay and in-hospital mortality) and ii) identify factors associated with IHC in women with T2DM who underwent breast cancer surgical procedures.

\section{METHODS}

We performed a retrospective, observational study using the SNHDD, which is managed by the Spanish Ministry of Health, Social Services and Equality (MHSSE) and compiles all public and private hospital data covering $>98 \%$ of hospital admissions. ${ }^{8}$

The SNHDD was implemented in 1987. According to Spanish legislation, all public and private Spanish hospitals must periodically submit data to the health authorities regarding those patients who have been hospitalised for at least 24 hours. The information required includes patient's characteristics such as their clinical history number, personal ID number, date of birth, sex, country of birth and address. Clinical variables include: admission and discharge dates, up to 14 diagnoses at discharge and up to 20 procedures performed during the hospital stay. The primary/main diagnosis is defined as the condition which, after proper investigation, is considered the reason why the patient was admitted to the hospital. The secondary diagnosis includes those diseases or risk factors that coexist with the primary diagnosis at the time of admission or were detected during the hospitalisation and that, in the opinion of the treating physician, may have affected the patient's progress or treatment plan.

Procedures include those diagnostic or therapeutic procedures conducted during hospitalisation. Information on the service where the patient has received care and the type of discharge (home, decease, voluntary discharge, other hospital and social institution) is also collected.

All patients discharged from the hospital must have a discharge report signed by the physician discharging the patient that includes the above noted information. The Codification Unit of the hospital uses this discharge report and any additional information required by the hospital databases to complete the SNHDD. The database uses the International Classification of Diseases, Ninth Revision, Clinical Modification (ICD-9-CM) for coding.

The Spanish Ministry of Health conducts regular audits to assess the accuracy of the SNHDD. ${ }^{89}$ Several studies have been conducted to assess the validity of the SNHDD for several conditions, including diabetes. ${ }^{10-13}$

In Spain, the diagnosis of type 2 diabetes is mainly performed in primary care centres using the American Diabetes Association (ADA) Consensus Statement. ${ }^{14}$ Even if patients are diagnosed during hospitalisation, they are sent to their primary care centre for the follow-up and necessary medical prescriptions.

We analysed data collected between 1 January 2013 and 31 December 2014 for women with and without T2DM who were aged over 40 years. In both groups, we selected patients with a primary diagnosis of breast cancer (ICD-9-CM codes: 174.0-174.9) and whose medical procedures included BCS (ICD-9-CM codes: 85.20-85.25) and/ or mastectomy (ICD-9-CM codes: 85.41-85.48) in any procedure field of the database. If both types of procedures were recorded, that case was excluded $(n=137)$.

We identified 41458 admissions with breast cancer who had undergone surgical procedures in 2013 and 2014 in Spain. Among those admissions, 3882 (9.23\%) had T2DM. Patients who underwent surgical breast cancer procedures in the non-diabetes group were selected to create a control group. Cases were matched with controls by age and province of residence $(n=50)$; if more than one control was available for a case, the selection was conducted randomly. Ultimately, we identified 3826 pairs of women (98.6\% of cases matched). 
In the second phase, we repeated the process and selected women without diabetes matched by age, province of residence, type of procedure and stage. Therefore, we matched 1938 women with T2DM who underwent BCS and 1480 who underwent mastectomy (88\% of cases matched). Clinical characteristics included data on overall comorbidities at the time of diagnosis, which were assessed by calculating the Charlson Comorbidity Index (CCI) and excluding both cancer and diabetes as diseases. ${ }^{15}$ We divided patients into three categories: CCI0, as in those patients with no previously recorded disease; CCI1, patients with one disease category and CCI $\geq 2$, patients with two or more disease categories.

We retrieved data concerning specific comorbidities, including acute myocardial infarction, congestive heart failure, renal disease, stroke/transient ischemic attack (TIA)/thromboembolism (TEP) and vascular disease, as described by Quan et al and that were applied to ICD-9-CM. ${ }^{16}$

The tumour stage was classified as local (within the breast), regional (affecting the lymph nodes-primarily those in the armpit and/or upper arm) or distant (in other parts of the body) according to Escribà et al using the enhanced ICD-9-CM. ${ }^{3}$

Specific risk factors considered in the data analysis included obesity (ICD-9-CM code: 278.xx), hypertension (ICD-9-CM codes: 401; 401.0; 401.1; 401.9) and current smoking (ICD-9-CM codes: 305.1;V15.82) recorded during hospitalisation for breast cancer in any diagnostic position.

Additionally, we specifically recorded the following procedures: sentinel lymph node dissection (ICD-9-CM codes: $40.11 ; 40.19 ; 40.23)$ and axillary lymph node dissection (ICD-9-CM codes: 40.3; 40.50; 40.51).

Irrespective of the position at diagnosis or the procedure coding list, we retrieved data regarding in-hospital 'infectious complications' such as pneumonia (ICD-9-CM codes: 997.39; 480-488; 507.0-507.8), sepsis (ICD-9-CM codes: 995.91, 995.92) and surgical site infection (breast-specific infection, ICD-9-CM codes 611.0; postoperative infection, ICD-9-CM codes 998.5-998.59; cellulitis, ICD-9-CM codes 682.2, 682.9; Staphylococcus aureus, ICD-9-CM codes 041.1-041.19; incision/drainage, ICD-9-CM codes 85.0, 85.91. 83.44-83.49, 86.01, 86.04, $86.09,86.22,86.28)$. Additionally, we noted 'non-infectious complications' after surgery, which included postoperative haemorrhage/haematoma (ICD-9-CM codes 998.11, 998.12), fat necrosis (ICD-9-CM codes 567.82, 611.3), dehiscence (ICD-9-CM codes 875.0, 875.1, 879.0, 879.1, 998.3, 998.32) and necrosis (ICD-9-CM codes 998.83).

Hospital outcome variables included the length of hospital stay (LOHS) and in-hospital mortality (IHM), the latter being defined as the percentage of patients who died during the admission.

\section{Statistical analysis}

A descriptive statistical analysis was performed for all continuous variables and categories by stratifying admissions for breast cancer according to diabetes status. Variables were expressed as proportions, either as the means with SD or medians with IQRs (LOHS). We performed bivariate conditional logistic regression models to compare the prevalence of clinical characteristics, risk factors, comorbidities, procedures, complications and in-hospital outcomes between patients with T2DM and controls. The analysis was conducted for the entire sample and stratified according to the procedure type (ie, BCS or mastectomy).

To identify variables associated with IHC among patients after breast cancer surgical procedures and T2DM, we performed a logistic regression analysis with any complication (infectious or non-infectious) as a binary outcome variable. Finally, we performed two logistic regression analyses to specifically identify variables associated with complications among patients suffering from diabetes who underwent BCS and mastectomy. The variables included in these models were those with significant results in the bivariate analysis $(\mathrm{p}<0.05)$ and those considered relevant in other investigations. The variables included in these models were age, CCI, stage and obesity. The remaining variables were not significant in the bivariate analysis.

Estimates were described as the OR with associated 95\% CIs.

The matching of cases with controls and all statistical analyses were performed using Stata V.10.1 (Stata, College Station, Texas, USA). Statistical significance was set at $\mathrm{p}<0.05$ (two-tailed).

\section{Ethical aspects}

Data confidentiality was maintained at all times, in accordance with Spanish law. Given the anonymous and mandatory nature of the dataset, the requirement for informed consent was deemed unnecessary.

\section{RESULTS}

Before matching was conducted, women with T2DM (3882) were significantly older ( $70.66 \pm 10.24$ vs $60.2 \pm 12.71$ years; $\mathrm{p}<0.001)$ than women without T2DM (37 576).

Regarding the procedures that we analysed, when we compared women with T2DM with matched controls by age and province of residence, significant differences $(p=0.04)$ were detected, with a higher incidence of mastectomy in women with T2DM compared with those without diabetes $(44.69 \%$ vs $42.42 \%, \mathrm{p}<0.05)$ and a greater rate of BCS in patients without T2DM $(57.58 \%$ vs $55.31 \%)$.

In contrast, we found that the local tumour stage of the disease was more prevalent in women without T2DM than in those with T2DM (73.86\% vs $71.33 \%$; $\mathrm{p}=0.020)$.

Table 1 shows the characteristics of hospital admissions for patients with breast cancer who underwent a surgical procedure and who underwent BCS procedures and 


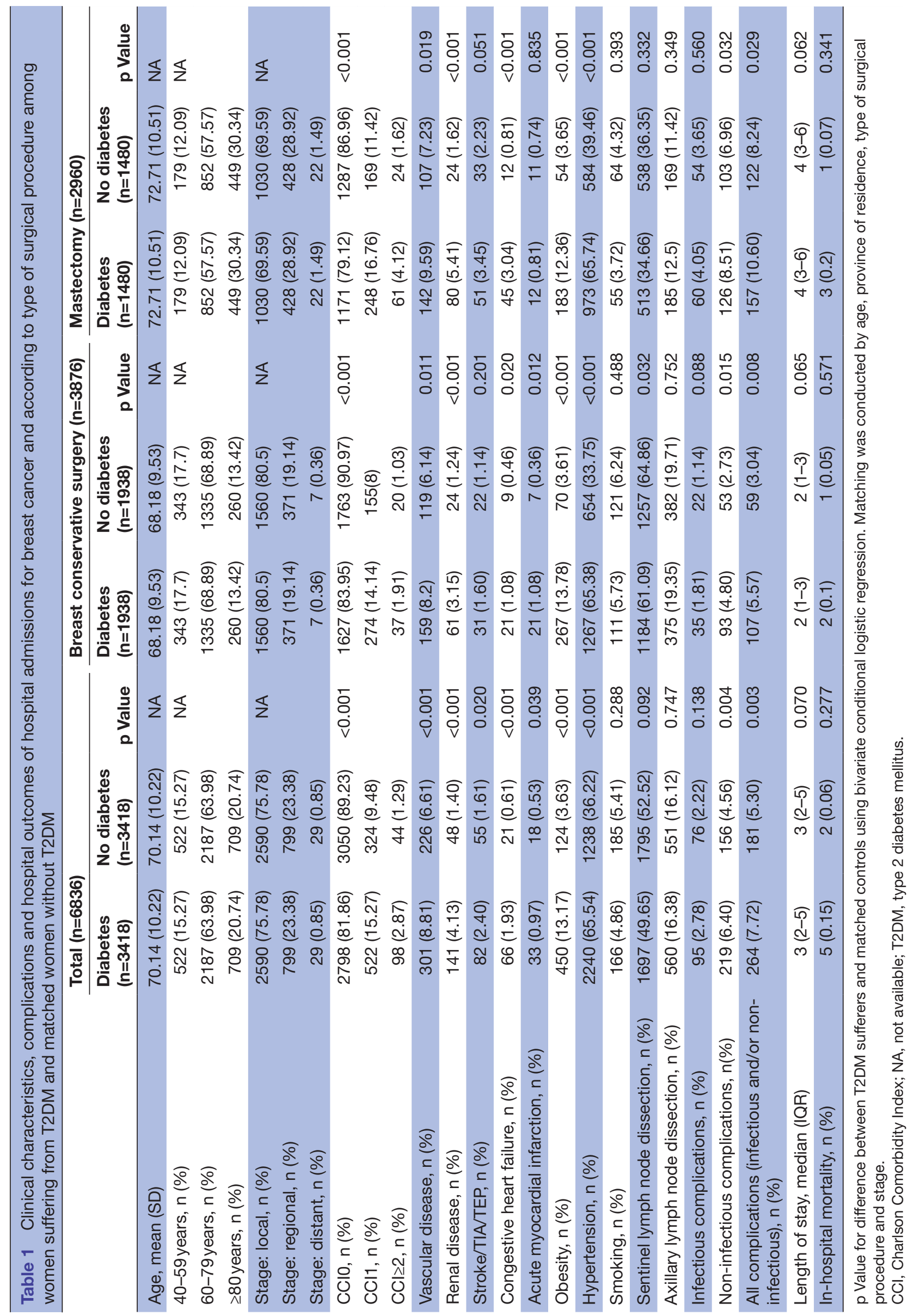


mastectomy among women with T2DM and age-matched controls without T2DM.

When we compared women with T2DM with matched controls, by age, province of residence and tumour stage, we found that patients with diabetes had more coexisting medical conditions according to the CCI $(p<0.001)$. Specifically, women with T2DM had a higher prevalence of vascular disease $(8.81 \%$ vs $6.61 \%)$, renal disease $(4.13 \%$ vs $1.40 \%)$, stroke $(2.40 \%$ vs $1.61 \%)$, congestive heart failure $(1.93 \%$ vs $0.61 \%)$ and acute myocardial infarction $(0.97 \%$ vs $0.53 \%)$. Additionally, the prevalence of hypertension ( $65.54 \%$ vs $36.22 \%$ ) and obesity $(13.17 \%$ vs $3.63 \%$ ) was higher in patients with T2DM.

As shown in table 1, all types of IHC were more frequent among women with T2DM $(7.72 \%)$ than in women without diabetes $(5.30 \% ; \mathrm{p}=0.003)$. When the types of complication were analysed, only non-infectious complications showed significant differences $(6.40 \%$ vs $4.56 \%)$.

Median LOHS and IHM values did not differ between women with and without diabetes.

As can be seen in table 1 , women with diabetes who underwent BCS were significantly younger than those who underwent a mastectomy (68.18 vs 72.71 years; $\mathrm{p}<0.001)$.

Among women who underwent a BSC, those with T2DM had higher frequencies of comorbidities according to the CCI, when compared with matched controls $(p<0.001)$. Vascular disease, renal disease, acute myocardial infarction, obesity and hypertension were significantly more prevalent in patients with diabetes than in matched controls. We found a significantly lower rate of sentinel lymph node dissection in patients with T2DM compared with controls $(61.09 \%$ vs $64.86 \%$; $\mathrm{p}=0.032)$.

Women with T2DM who underwent a BSC had a higher prevalence of all IHC (infectious and/or non-infectious) compared with those without diabetes $(5.57 \%$ vs $3.04 \%$, respectively, $\mathrm{p}=0.008)$ and also for non-infectious complications alone $(4.80 \%$ vs $2.73 \%)$.
Women with T2DM who underwent mastectomy had more coexisting medical conditions according to the CCI and a higher prevalence of vascular disease, renal disease, congestive heart failure, obesity and hypertension than women without diabetes. Additionally, women with T2DM had a higher prevalence of IHC (infectious and/ or non-infectious) than those without diabetes $(10.60 \%$ vs $8.24 \%$, respectively; $\mathrm{p}=0.029)$. According to the type of complications, differences were only significant for non-infectious complications ( $8.51 \%$ vs $6.96 \%$; $\mathrm{p}=0.032)$.

Table 2 shows the results of logistic regression analyses to assess those factors associated with IHC in patients with T2DM during hospital admission for breast cancer who underwent any breast surgical procedures and, more specifically, according to the type of procedure. Among women with T2DM with breast cancer and after any surgical procedure (ie, conservative or mastectomy), comorbidity (vs no comorbidites, OR $1.72,95 \%$ CI 1.30 to 2.27 for one comorbidity; OR $2.55,95 \%$ CI 1.55 to 4.17 for two or more comorbidities) and obesity (OR 1.54, 95\% CI 1.14 to 2.07 ) were the factors most strongly associated with a higher rate of IHC. These two factors also increased the risk of IHC for women who underwent BCS. However, among those who underwent mastectomy, only comorbidity was associated with a higher rate of IHC.

\section{DISCUSSION}

T2DM is considered to be a common comorbidity that is present in many patients with breast cancer, correlating with poor clinical outcomes. ${ }^{17} 18$ According to information obtained from the SNHDD, compared with non-diabetic counterparts, women with T2DM undergoing surgical breast cancer procedures have more risk factors, comorbidities and advanced cancer presentations.

Several studies have reported that the presence of risk factors, such as hypertension and obesity, are common

Table 2 Logistic regression analysis of the factor associated with in-hospital complications in women with type 2 diabetes during hospital admission for breast cancer according to the type of surgical procedure

\begin{tabular}{|c|c|c|c|c|}
\hline & & $\begin{array}{l}\text { Any breast cancer surgical procedure } \\
\text { (conservative or mastectomy) } \\
\text { OR }(95 \% \mathrm{Cl})\end{array}$ & $\begin{array}{l}\text { Breast conservative } \\
\text { surgery } \\
\text { OR }(95 \% \mathrm{Cl})\end{array}$ & $\begin{array}{l}\text { Mastectomy } \\
\text { OR (95\% CI) }\end{array}$ \\
\hline \multirow{3}{*}{$\begin{array}{l}\text { Age groups, } \\
\text { years }\end{array}$} & $40-59$ & 1 & 1 & 1 \\
\hline & $60-79$ & $0.98(0.70$ to 1.37$)$ & 0.79 (0.50 to 1.26$)$ & 1.12 (0.67 to 1.86$)$ \\
\hline & $\geq 80$ & $1.16(0.79$ to 1.71$)$ & $0.92(0.50$ to 1.67$)$ & 1.22 (0.71 to 2.10$)$ \\
\hline \multirow[t]{3}{*}{$\mathrm{CCl}$} & 0 & 1 & 1 & 1 \\
\hline & 1 & 1.72 (1.30 to 2.27 ) & 1.75 (1.12 to 2.71$)$ & 1.64 (1.14 to 2.35$)$ \\
\hline & $\geq 2$ & 2.55 (1.55 to 4.17 ) & 3.33 (1.48 to 7.48 ) & 2.10 (1.13 to 3.91$)$ \\
\hline \multirow[t]{2}{*}{ Stage } & Local & 1 & 1 & 1 \\
\hline & Regional/distant & 1.21 (0.98 to 1.48$)$ & $1.08(0.74$ to 1.55$)$ & 1.19 (0.93 to 1.52$)$ \\
\hline Obesity & Yes & 1.54 (1.14 to 2.07 ) & 1.68 (1.07 to 2.60$)$ & 1.46 (0.98 to 2.19$)$ \\
\hline
\end{tabular}

All variables included in the models are shown.

$\mathrm{CCl}$, Charlson Comorbidity Index. 
in both patients with diabetes and those with breast cancer. ${ }^{19} 20$

In our current study, women with T2DM were significantly more likely to suffer from advanced-stage breast cancer when compared with women without diabetes. Our findings are consistent with other studies demonstrating a more advanced stage of breast cancer among women with diabetes. ${ }^{21}{ }^{22}$ A study conducted in Canada showed that diabetes was associated with more advanced-stage breast cancer, even after accounting for differences in screening mammogram use and other factors. ${ }^{23}$ There are several possible explanations for this association, including the fact that mammograms are less sensitive for the detection of early lesions in women with T2DM because of higher rates of obesity. ${ }^{24}$ Finally, tumours may progress more rapidly in patients with diabetes, or diabetes may lead to a higher metastatic potential. ${ }^{25}$

We observed that mastectomies are performed more frequently on diabetic women, which could be explained by several factors, including higher comorbidity, higher stage disease at diagnosis, the presence of more biologically aggressive tumours and the fact that diabetes is a major risk factor in breast cancer recurrence. ${ }^{325}$ Kaplan et al examined 483 patients with breast cancer who had undergone a mastectomy between 1998 and 2010. They concluded that the recurrence of breast cancer was significantly increased in patients with diabetes (OR 2.21; $95 \%$ CI 1.23 to $3.96 ; p=0.008) .{ }^{26}$

As expected, we observed that in-hospital mortality and IHC rates in breast cancer surgery were low. This finding is consistent with de Blacam et al, who, in a multi-institutional study of patients undergoing mastectomy and BCS in the USA, found that the overall 30-day morbidity rate was $5.6 \%$ for all procedures. ${ }^{7}$

We found that women with T2DM who underwent breast cancer surgical procedures had a higher frequency of overall IHC compared with women without diabetes. Several studies have reported that diabetes is a risk factor for surgical site infections after breast operations. ${ }^{7}$ A systematic review/meta-analysis concluded that patients with diabetes were more vulnerable to surgical site infections after breast cancer surgery (OR 1.88; 95\% CI 1.47 to 2.39$){ }^{27}$

In this study, comorbidity was a strong predictor of IHC in women with T2DM who underwent surgical breast cancer-related procedures. Dehal et al studied the impact of comorbidity using hospital discharge data from the 'Healthcare Utilisation Project: Nationwide In-patient Sample' database in women with a primary diagnosis of breast cancer after undergoing breast surgery. They found that women with a severe (CCI score $\geq 3$ ) comorbidity were 4.6 times more likely to develop a postoperative complication when compared with patients without a comorbidity. ${ }^{28}$

In our present study, obesity was a predictor of IHC in women with diabetes who underwent BCS. Being overweight or obese has been associated with higher rates of treatment-related sequelae, such as lymphedema, fatigue and arthralgia. ${ }^{29}$ Helyer et al concluded that obesity is a risk factor for the development of postoperative lymphedema in patients with breast cancer (OR 1.8; 95\% CI 1.0004 to 1.165$){ }^{30}$ Excess adiposity may increase the risk of lymphedema by driving increased inflammation, adding stress to the lymphatic system or slowing postsurgical healing times. ${ }^{31}$ However, as will be commented in the limitations section the uncertainty regarding the validity of the obesity codification in the SNHDD requires caution when interpreting this result.

The strengths of our study included its large simple size, the standardised methodology, and use of age-matched and province-matched patients with no T2DM to control for the confounding effects of these variables. However, our study has several limitations. First, the validity of the T2DM must be discussed. A study conducted to validate the diabetes mellitus diagnosis in the computerised clinical records of primary healthcare in Spain, taking the 2003 ADA Consensus Statement as the gold standard, found that the agreement was very high $(\kappa=0.990)$, with a specificity of $99.49 \%$ and a sensitivity of $99.53 \% .{ }^{14}$ The validity of the diabetes diagnosis in the SNHDD has been assessed in two previous studies, revealing a sensitivity of $55 \%$ and $63.7 \%$ and a specificity of approximately $97 \% .^{1011}$

The only moderate sensitivity found means that an important proportion of patients with T2DM do not have this diagnosis codified in their discharge report. On the other hand, the very high specificity means that most patients without a T2DM diagnosis do not really have this disease; therefore, we think that the effect of this misclassification on our design is possibly very small. Furthermore, the database was designed for administrative rather than research purposes, therefore conditions such as obesity, infection, hypertension and smoking may not have been adequately recorded in the database. 81011

The validity of the variable 'obesity' in our investigation must be discussed. In our study population, the prevalence of obesity is very low among women with diabetes, which is possibly a consequence of under-recording this condition. Previous studies conducted in Spain and other countries have also found an under-reporting of obesity in administrative data. ${ }^{32-35}$ These authors suggest the following possible reasons to explain the low rates of obesity in administrative data: i) obesity is not explicitly mentioned in physician reports; ii) people who codify may not record obesity owing to time constraints when performing data abstraction; iii) when time for coding is limited, coders tend to include severe conditions but not risk factors and iv) the diagnosis of obesity is often not based on the body mass index (BMI), but rather on the subjective observation made by the clinician, which means that more severe obesity is overcodified. ${ }^{33-35}$ Furthermore, a possible differential information bias may occur and thus the misclassification of obesity may be related to the presence of diabetes. Thus, ICD codes for obesity may be more commonly assigned to patients suffering from other comorbidities (including diabetes) 
or postoperative complications. This suggests a greater association between obesity and adverse events than what is obtained based on the BMI calculations. ${ }^{335}$ As a result, any association between obesity and the presence of IHC must be interpreted with caution.

Second, unfortunately the ICD-9 does not include information regarding whether a condition is part of the patient's past medical history or if this appeared during the hospitalisation. Therefore, it is possible that a patient may first be diagnosed with diabetes or any other condition studied during the hospitalisation. However, we think that this possibly affects a very small proportion of patients and thus the effect on our results would be minimal.

Third, the database that we used contained no information about chemotherapy or radiotherapy treatments prior to surgery, which may have affected our outcome variables.

Fourth, we lacked data such as breast cancer characteristics or the time span since diagnosis.

Finally, other relevant variables such as laboratory results, BMI, medical treatments (eg, glucose-lowering drugs, insulin or antibiotics), patients who have undergone a prosthetic reconstruction and the eventual use of an acellular dermal matrix are not included in the SNHDD.

\section{CONCLUSIONS}

We conclude that women with T2DM who undergo surgical procedures to treat breast cancer have more comorbidities, risk factors and a more advanced cancer presentation than women without T2DM. Mastectomies are more frequently performed in diabetic women. Moreover, procedures carried out in women with T2DM were associated with greater IHC. Finally, comorbidity was a strong predictor of IHC in women with T2DM.

\section{Author affiliations \\ ${ }^{1}$ Department of Medicine and Surgery, Psychology, Preventive Medicine and Public Health, Medical Microbiology and Immunology, Nursing and Oral Medicine, Universidad Rey Juan Carlos, Alcorcón, Spain \\ ${ }^{2}$ Respiratory Care Department, Hospital General Universitario Gregorio Marañón, Madrid, Spain \\ ${ }^{3}$ Internal Medicine Department, Hospital General Universitario Gregorio Marañón, Madrid, Spain \\ ${ }^{4}$ Internal Medicine Department, Hospital General Universitario Gregorio Marañón, Madrid, Spain \\ ${ }^{5}$ Health Security Agency, Ministry of Health, Social Services and Equality, Madrid, Spain \\ ${ }^{6}$ Subdirección General de Investigación Sanitaria, Madrid, Spain}

Acknowledgements The authors would like to thank the Spanish Ministry of Health and Social Policy for providing the records of the SNHDD (Conjunto Mínimo Básico de Datos).

Contributors AL-de-A and RJ-G participated in the conception and design, analysis, interpretation and writing of the article. VH-B and IJ-T contributed their statistical expertise and towards conception and design, interpretation and writing of the article. JdeM-D, MM-B, JMdeM-Y, NP-F, MAS-F, JLdeB and MR-M were involved in the critical revision of the article, interpretation and design. All authors have seen and approved the final version.
Funding This study is part of research funded by the FIS (Fondo de Investigaciones Sanitarias-Health Research Fund, grants no. PI13/00118 and PI16/00564, Instituto de Salud Carlos III) co-financed by the European Union through the Fondo Europeo de Desarrollo Regional (FEDER, 'Una manera de hacer Europa') and by the Grupo de Excelencia Investigadora URJC-Banco Santander N³0VCPIGI03: Investigación traslacional en el proceso de salud-enfermedad (ITPSE).

Competing interests None declared.

Ethics approval The study protocol was approved by the Ethics Committee of the Rey Juan Carlos University.

Provenance and peer review Not commissioned; externally peer reviewed.

Data sharing statement No additional data are available.

Open Access This is an Open Access article distributed in accordance with the Creative Commons Attribution Non Commercial (CC BY-NC 4.0) license, which permits others to distribute, remix, adapt, build upon this work non-commercially, and license their derivative works on different terms, provided the original work is properly cited and the use is non-commercial. See: http://creativecommons.org/ licenses/by-nc/4.0/

(c) Article author(s) (or their employer(s) unless otherwise stated in the text of the article) 2017. All rights reserved. No commercial use is permitted unless otherwise expressly granted.

\section{REFERENCES}

1. Fitzmaurice C, Allen C, Barber RM, et al. Global Burden of Disease Cancer Collaboration. Global, Regional, and National Cancer Incidence, Mortality, Years of Life Lost, Years Lived With Disability, and Disability-Adjusted Life-years for 32 Cancer Groups, 1990 to 2015: A Systematic Analysis for the Global Burden of Disease Study. JAMA Oncol 2017;3:524-48.

2. Raine $\mathrm{R}$, Wong $\mathrm{W}$, Scholes $\mathrm{S}$, et al. Social variations in access to hospital care for patients with colorectal, breast, and lung cancer between 1999 and 2006: retrospective analysis of hospital episode statistics. BMJ 2010;340:b5479.

3. Escribà JM, Pareja L, Esteban L, et al. Trends in the surgical procedures of women with incident breast cancer in Catalonia, Spain, over a 7-year period (2005-2011). BMC Res Notes 2014;7:587.

4. Chang JM, Kosiorek HE, Dueck AC, et al. Trends in mastectomy and reconstruction for breast cancer; a twelve year experience from a tertiary care center. Am J Surg 2016;212:1201-10.

5. Pyfer B, Chatterjee A, Chen L, et al. Early postoperative outcomes in breast conservation surgery versus simple mastectomy with implant reconstruction: a NSQIP analysis of 11,645 patients. Ann Surg Oncol 2016:23:92-8.

6. El-Tamer MB, Ward BM, Schifftner T, et al. Morbidity and mortality following breast cancer surgery in women: national benchmarks for standards of care. Ann Surg 2007;245:665-71.

7. de Blacam C, Ogunleye AA, Momoh AO, et al. High body mass index and smoking predict morbidity in breast cancer surgery: a multivariate analysis of 26,988 patients from the national surgical quality improvement program database. Ann Surg 2012;255:551-5.

8. Ministerio de Sanidad, Servicios Sociales e Igualdad. Registro de altas de los hospitales generales del sistema nacional de salud: CMBD. https://www.msssi.gob.es/estadEstudios/estadisticas/ cmbdhome.htm. (accessed 30 Aug 2017).

9. Estudio de las comorbilidades que componen el índice de Elixhauser. Análisis de prevalencia y fiabilidad en los registros del CMBD estatal de hospitalizacion. http://icmbd.es/docs/informe-comorbilidades-1. pdf. (Accessed 30 August 2017).

10. Ribera A, Marsal JR, Ferreira-González I, et al. Predicting in-hospital mortality with coronary bypass surgery using hospital discharge data: comparison with a prospective observational study. Rev Esp Cardiol 2008:61:843-52.

11. Rodrigo-Rincón I, Martin-Vizcaíno MP, Tirapu-León B, et al. Usefulness of administrative databases for risk adjustment of adverse events in surgical patients. Cir Esp 2016;94:165-74.

12. Calle JE, Saturno PJ, Parra P, et al. Quality of the information contained in the minimum basic data set: results from an evaluation in eight hospitals. Eur J Epidemiol 2000;16:1073-80.

13. Hernández Medrano I, Guillán M, Masjuan J, et al. Reliability of the minimum basic dataset for diagnoses of cerebrovascular disease. Neurologia 2017;32:74-80.

14. de Burgos-Lunar C, Salinero-Fort MA, Cárdenas-Valladolid J, et al. Validation of diabetes mellitus and hypertension diagnosis in 
computerized medical records in primary health care. BMC Med Res Methodol 2011;11:146.

15. Charlson ME, Pompei P, Ales KL, et al. A new method of classifying prognostic comorbidity in longitudinal studies: development and validation. J Chronic Dis 1987;40:373-83.

16. Quan H, Sundararajan V, Halfon $P$, et al. Coding algorithms for defining comorbidities in ICD-9-CM and ICD-10 administrative data. Med Care 2005;43:1130-9.

17. Lipscombe LL, Goodwin PJ, Zinman B, et al. The impact of diabetes on survival following breast cancer. Breast Cancer Res Treat 2008;109:389-95.

18. Peairs KS, Barone BB, Snyder CF, et al. Diabetes mellitus and breast cancer outcomes: a systematic review and meta-analysis. J Clin Oncol 2011;29:40-6.

19. Patterson RE, Flatt SW, Saquib N, et al. Medical comorbidities predict mortality in women with a history of early stage breast cancer. Breast Cancer Res Treat 2010;122:859-65.

20. Jousheghany F, Phelps J, Crook T, et al. Relationship between level of HbA1C and breast cancer. BBA Clin 2016;6:45-8.

21. Srokowski TP, Fang S, Hortobagyi GN, et al. Impact of diabetes mellitus on complications and outcomes of adjuvant chemotherapy in older patients with breast cancer. J Clin Oncol 2009;27:2170-6.

22. van de Poll-Franse LV, Houterman S, Janssen-Heijnen ML, et al. Less aggressive treatment and worse overall survival in cancer patients with diabetes: a large population based analysis. Int $J$ Cancer 2007;120:1986-92.

23. Lipscombe LL, Fischer HD, Austin PC, et al. The association between diabetes and breast cancer stage at diagnosis: a population-based study. Breast Cancer Res Treat 2015;150:613-20.

24. Kerlikowske K, Walker R, Miglioretti DL, et al. Obesity, mammography use and accuracy, and advanced breast cancer risk. J Natl Cancer Inst 2008;100:1724-33.
25. Godsland IF. Insulin resistance and hyperinsulinaemia in the development and progression of cancer. Clin Sci 2010;118:315-32.

26. Kaplan MA, Pekkolay Z, Kucukoner M, et al. Type 2 diabetes mellitus and prognosis in early stage breast cancer women. Med Oncol 2012;29:1576-80.

27. Xue DQ, Qian C, Yang L, et al. Risk factors for surgical site infections after breast surgery: a systematic review and meta-analysis. Eur $J$ Surg Oncol 2012;38:375-81.

28. Dehal A, Abbas A, Johna S. Comorbidity and outcomes after surgery among women with breast cancer: analysis of nationwide in-patient sample database. Breast Cancer Res Treat 2013;139:469-76.

29. Ridner SH, Dietrich MS, Stewart BR, et al. Body mass index and breast cancer treatment-related lymphedema. Support Care Cancer 2011;19:853-7.

30. Helyer LK, Varnic M, Le LW, et al. Obesity is a risk factor for developing postoperative lymphedema in breast cancer patients. Breast J 2010;16:48-54.

31. Ahmed RL, Schmitz KH, Prizment AE, et al. Risk factors for lymphedema in breast cancer survivors, the lowa Women's Health Study. Breast Cancer Res Treat 2011;130:981-91.

32. Sánchez-Muñoz LA. Nutritional status, heart failure and minimum basic data set. Rev Esp Cardiol 2012;65:583.

33. Martin BJ, Chen G, Graham M, et al. Coding of obesity in administrative hospital discharge abstract data: accuracy and impact for future research studies. BMC Health Serv Res 2014;14:70.

34. Lujic S, Watson DE, Randall DA, et al. Variation in the recording of common health conditions in routine hospital data: study using linked survey and administrative data in New South Wales, Australia. BMJ Open 2014;4:e005768.

35. McLynn RP, Geddes BJ, Cui JJ, et al. Inaccuracies in ICD coding for obesity would be expected to bias administrative database spine studies toward overestimating the impact of obesity on perioperative adverse outcomes. Spine 2017:1. 